OPEN ACCESS

Edited by:

Michele Marchesi,

University of Cagliari, Italy

Reviewed by:

Andreas de Blanche,

University West, Sweden

Nicola Dimitri,

University of Siena, Italy

*Correspondence:

Christian Stummer

christian.stummer@uni-bielefeld.de

Specialty section:

This article was submitted to Blockchain Economics,

a section of the journal

Frontiers in Blockchain

Received: 14 December 2020

Accepted: 01 March 2021

Published: 18 March 2021

Citation:

Klein M and Stummer C (2021)

Feeless Micropayments as Drivers for New Business Models: Two

Exemplary Application Cases.

Front. Blockchain 4:641508.

doi: 10.3389/fbloc.2021.641508

\section{Feeless Micropayments as Drivers for New Business Models: Two Exemplary Application Cases}

\author{
Marvin Klein and Christian Stummer* \\ Faculty of Business Administration and Economics, Bielefeld University, Bielefeld, Germany
}

Upcoming cryptocurrency approaches claim to enable feeless micropayments. Assuming that this promise will be kept, this is likely to give rise to new business models that entail transactions worth fractions of a cent. In this mini review, we describe two exemplary application cases and outline the associated research activities for each. The first case considers subscription models in consumer markets for which feeless micropayments might be used to expand and, thus, enrich the range of plans by offering shorter subscription times. The second case is concerned with prosumers who might be willing to sell self-generated data (e.g., data generated through their smart meters).

Keywords: cryptocurrency, micropayments, business models, subscription model, self-generated data

\section{INTRODUCTION}

Imagine buying a new car for $\$ 10,000$ and during the payment process, the seller charges you an extra $\$ 1,000$ for the money transfer. This appears to have the makings of a scam and seems like something that must not be allowed to happen in the real world. Nonetheless, relatively speaking, this is how PayPal charges customers for (commercially) transferring $\$ 1$ within the United States-namely, 10 cents (PayPal, 2021). As the fee is relatively high compared to the transferred value itself, digital micropayments are often unattractive for the seller. Yet, micropayments are common in the online world, for example, when paying for digital content such as news articles, songs, movies, apps, or in-game purchases. Nowadays, sellers attempt to bundle multiple transactions in order to reduce such fees, for example, by means of aggregation of payments, prepay systems, or subscription contracts (Kaufman et al., 2011; Turban et al., 2018).

New distributed ledger technology (DLT) approaches may considerably reduce transaction costs. While traditional blockchain-based DLTs-like Bitcoin-have relatively high transaction fees, which makes them, in their current form, unsuitable for micropayments, several possible solutions for addressing this problem are already proposed, including the introduction of second layers (e.g., Lightning Network) or increasing the block size; for an overview see Kuśzmierz et al. (2021). Further promising DLT approaches that could serve as a means for feeless micropayments are not based on a blockchain but on a directed acyclic graph (DAG). Thus, DLTs such as NANO (LeMahieu, 2018) or IOTA (Popov, 2019) do not require the involvement of the so-called miners. A prime example of a DAG is IOTA's Tangle, in which every new transaction is required to reference 
exactly two previous transactions (Gal, 2018; Popov, 2018). To be fair, DAGs still rely on nodes that consume a minimum amount of energy and their users require an internet connection, a suitable device (e.g., smart phone), and energy to run it; moreover, as spam prevention, a minimum amount of proof-ofwork is required, for which a midrange smart phone consumes approximately $0.00016 \mathrm{kWh}$ (Sori et al., 2020). To put it in a nutshell, executing a DAG transaction technically is not totally free of costs, but costs are negligible (i.e., comparable to sending an email).

Although these novel DLT approaches have not yet been widely implemented in practice, for the purpose of this research, we assume that one of them will succeed and the promise of (almost) feeless micropayments can be kept. If so, we expect to see both adjustments for subscription models and opportunities for the development of new business models arising from the possibility of making transfer payments that are worth even less than a cent. The associated relevance of such approaches for digital transformation is obvious, as feeless micropayments will further this transformation. In a more distant future, DLT-based approaches might also be used for creating smart contracts or digital identities (World Economic Forum, 2020), all of which would likely give rise to interesting follow-up research questions.

The contribution of the mini review at hand is twofold. First, we strive to draw attention to the potential of new DLT approaches that enable feeless micropayment as drivers for both adaption of existing business models and creating new ones. Second, we illustrate corresponding business models by means of two exemplary cases and outline the subsequent research activities.

\section{THE CASE OF EXPANDING THE CURRENT SUBSCRIPTION MODELS}

Once feeless micropayments become widely accessible, they will make the bundling of several small transactions into a bigger one obsolete. Their impact on the prepaid system and on subscription contracts is less obvious because service providers can benefit from these payment modes also in some other ways. In the case of prepayment, consumers advance money, and many of them might even never use up their deposit (Cerullo, 2020). In the subscription case, providers have an opportunity to learn about who is using their service and about their customers' interests. Moreover, certain consumers are willing to pay more for a flat rate than for services that are precisely metered (Odlyzko, 2003; Lambrecht and Skiera, 2006). Thus, some providers will stick with their existing business models while others might develop novel business models based on feeless micropayments as a means to generate additional revenue (i.e., as a supplement to their existing business model). In the following, we outline possible implications for current subscription models.

Today, numerous sellers utilize subscription models (e.g., online newspapers or media-service providers), in which customers pay a fixed (usually monthly) fee and receive unrestricted access to the content of a platform, regardless of how much they consume. With the possibility of transferring money without fees, content providers-such as Netflix, Spotify, or Sky-can expand the variety of their subscription plans by offering shorter subscription periods-such as a subscription for just one day or even only a single episode of a series. While, from the viewpoint of many vendors, today's payment systems (e.g., credit cards, paypal) are too expensive for processing the corresponding small payments (Turban et al., 2018), in the event of feeless micropayments, a content provider (e.g., Netflix) might more easily attempt to attract consumers who are already subscribed on a competing platform (e.g., Amazon Prime Video) and who would like to watch a specific series but do not wish to fully commit to another monthly contract. As the consumers' financial risk would be low, such an offer can overcome their adoption barrier and, after becoming familiar with the platform, they might even consume more content than originally planned-similar to the effect of free or discounted trial subscriptions (Cheng and Tang, 2010) and in accordance with prospect theory (Kahneman and Tversky, 1979). Eventually, such an expanded subscription model can both yield overall higher revenues for the seller and lead to more consumer satisfaction. Further application fields are, for example, customers paying for one specific article in an online newspaper (Graybeal and Hayes, 2011) or paying just for the time they watch a live sports event (e.g., the semifinal of a world cup match). To be fair, certain platforms (e.g., Amazon Prime Video) already offer customers the option to only pay for, for example, a specific series. However, such an offer is often more expensive than a subscription for the entire month with which consumers obtain access to considerably more content. Moreover, most often, an annoying and time-wasting registration and verification process is necessary. In a situation in which one spontaneously wants to watch the end of the above-mentioned match, this is probably a rather inconvenient user experience and, thus, inhibits such purchases.

Therefore, we consider this a promising research field in terms of pricing and behavioral economics. In particular, more insight might be sought in three regards. First, future research should aim to assess the possible gain in market potentialthat is, the number and frequency of consumers who actually face a situation in which they like to consume specific content but do not succeed because they lack the necessary account or subscription from the respective content provider. Second, the consumers' current "solution strategies" in such instances are of interest: Do they just set up a monthly subscription? Do they use a friend's account? Do they go to a friend to watch the series or the football match, etc. together? Do they resort to illegal means like using online pirate streaming sites? Or do they merely abstain from consuming the specific content? Third, consumers' willingness to pay for the possibility of obtaining short-period access to content should be examined.

\section{THE CASE OF SELLING SELF-GENERATED DATA}

Feeless value transactions will also enable new business models, for which we identified three particularly promising fields of 
application-namely, trading of small amounts of energy (e.g., electric vehicle charging; see Strugar et al., 2018), minimal usage of services or devices (e.g., platooning; see Fraunhofer Innovisions, 2019), and selling of self-generated data (e.g., traffic information; see Jaguar Land Rover, 2019). In the following account, we address the latter field.

The nucleus of corresponding business models is that most data is produced for a specific purpose but then remains untapped (Noll and Alt, 2020), although it may have someeven though miniscule-value for other purposes as well. Often, this data is individually owned by the many people who generated it and, with regulations like GDPR in place, cannot be freely used without their consent. Feeless micropayments might play a crucial role in making such data accessible to others who might be willing to pay for its usage, because such micropayments allow for trading these data pieces in an economically efficient manner.

Sensor data collected by a smart car is an illustrative example as data on traffic, potholes, or empty parking spots (Frederiksen, 2019; Jaguar Land Rover, 2019) could help other car drivers to save time and cost (and thereby also reduce CO2 emissions). Car owners would probably not object to selling this data, particularly if it would be anonymous and if they can profit from it in some manner. Note that in this example, the car drivers "producing" data could also "consume" data supplied by other drivers, which essentially makes them so-called prosumers (Toffler, 1980).

Another example is data generated by smart meters in households (Rooijakkers, 2018). Such data can be of value for local authorities in their effort to effectively strengthen electric infrastructure according to the received valid information on changes in power consumption patterns (e.g., due to an increased number of electric vehicles in a region). Researchers could require such data for a certain study, like investigating differences in cooking behavior in a population. Businesses would likely utilize such data patterns in order to offer individualized energy-supply contracts or to offer the replacement of a particularly energyinefficient home appliance. All these interested parties might be willing to pay (very small) amounts of money to each of the participating households for receiving this data and they could do so through feeless micropayments, preferably on a decentralized data marketplace (Fernandez et al., 2020). Again, consumers could turn into prosumers. They could purchase information regarding energy production in their region and temporarily switch to an alternative supplier; this supplier could even be a neighbor who has installed solar panels on the roof that currently have a surplus of energy because of numerous sunny days with strong sunshine. In such a case, feeless micropayments would effectively enable a trade of watthours. As a positive side effect, energy is not only utilized when it is produced but also where it is produced, which contributes to relieving the pressure on the overall electricity grid (Harbor, 2019).

However, there are different types of self-generated data that are considerably more sensitive, like health data produced by smart wearables (Becker et al., 2020). Therefore, future research should examine the extent to which consumers are willing to sell which type of personal data for which price.

\section{DISCUSSION}

The practical relevance of micropayments is supposed to substantially increase in the forthcoming years; with reference to micropayments among machines, experts expect that micropayments will have high impact and will be daily business in a few years (Schweizer et al., 2020). Feeless micropayments based on novel DLT protocols might adjust current modes of how to handle such micropayments and are likely to give rise to new business models based on the possibility to process micropayments. In order to assess the possible gain in market potential (in addition to the machine economy), future research activities should ascertain the number and frequency of consumers who actually face a situation in which they, for example, like to consume certain specific content but do not succeed because they lack the necessary subscription from the respective content provider. Another interesting research topic is concerned with consumers' current "solution strategies" to obtain the desired content in such instances (e.g., using a friend's account). Quantitative studies, such as choice-based conjoint experiments, could be used in order to examine consumer preferences on subscription frequency; in subsequent studies, additional markets (e.g., the live sports event streaming market and online newspapers) could be included.

With respect to the business model with self-generated data, further research should study the extent to which and with whom consumers are willing to share their personal data in exchange for monetary compensation (through micropayments) and the intrinsic benefit of having contributed to, for example, a more reasonable energy usage. Moreover, the extent to which such a business model might increase interest in smart products might be an interesting research question.

While we picked exemplary fields of application for feeless micropayments and dwelled on the possible impact of such micropayments on existing or new business models, we feel that there are numerous more interesting fields (e.g., charity, crowdfunding, tips, microfinancing, and gaming), all of which may constitute promising avenues for further research in DLT economics.

\section{AUTHOR CONTRIBUTIONS}

MK proposed the research and provided a first draft of the manuscript. CS supervised the process and substantially revised the first draft which included reorganization of the manuscript, providing additional paragraphs, and extending, or at least, editing, all other paragraphs; at the end, no place of the initial version has remained untouched. MK and CS worked together in revising and fine-tuning the final version of the manuscript. Both authors contributed to the article and approved the submitted version.

\section{FUNDING}

We acknowledge support for the publication costs by the Open Access Publication Fund of Bielefeld University. 


\section{REFERENCES}

Becker, M., Matt, C., and Hess, T. (2020). It's not just about the product: how persuasive communication affects the disclosure of personal health information. ACM SGIMIS Database 51, 37-50. doi: $10.1145 / 3380799.3380804$

Cerullo, M. (2020). Consumers Waste Up to \$3 Billion in Unspent Gift Cards a Year. Available online at: https://www.cbsnews.com/news/unused-gift-cardsadd-up-to-3-billion-annually (accessed February 17, 2021).

Cheng, H. K., and Tang, Q. (2010). Free trial or no tree trial: optimal software product design with network effects. Eur. J. Operation. Res. 205, 437-447. doi: 10.1016/j.ejor.2010.01.014

Fernandez, D., Futoransky, A., Ajzenman, G., Travizano, M., and Sarraute, C. (2020). Wibson protocol for secure data exchange and batch payments. arXiv [Preprint]. Available online at: https://arxiv.org/abs/2001.08832v2 (accessed February 17, 2021).

Fraunhofer Innovisions (2019). Sicher verbunden: Blockchain-Konzept ermöglicht Vorteilsausgleich im Platoon [in German]. Available online at: https://www. fraunhofer-innovisions.de/blockchain/sicher-verbunden (accessed February 13, 2021).

Frederiksen, C. E. (2019). IOTA Showcases Sustainable Energy Traceability at Powerhouse Energy Positive Building. Available online at: https: //blog.iota.org/iota-renewable- energy-transfer-at-powerhouse-smartbuilding-dd42bbf799e5 (accessed February 13, 2021).

Gal, A. (2018). The Tangle: An Illustrated Introduction. Available online at: https: //blog.iota.org/the-tangle-an-illustrated-introduction-4d5eae6fe8d4 (accessed February 13, 2021).

Graybeal, G. M., and Hayes, J. L. (2011). A modified news micropayment model for newspapers on the social web. Int. J. Med. Manag. 13, 129-148. doi: 10.1080/ 14241277.2011.568808

Harbor, C. (2019). ElaadNL Develops Autonomous Self-Balancing Power Grid Using IOTA. Available online at: https://blog.iota.org/elaadnl-develops-autonomousself-balancing-power-grid-using-iota-de52e9638548 (accessed February 13, 2021).

Jaguar Land Rover (2019). On the Money: Earn as You Drive with Jaguar Land Rover. Available online at: https://www.jaguarlandrover.com/news/2019/04/ money-earn-you-drive-jaguar-land-rover (accessed February 13, 2021).

Kahneman, D., and Tversky, A. (1979). Prospect theory: an analysis of decision under risk. Econometrica 47, 263-292. doi: 10.2307/1914185

Kaufman, S., Ramani, A., Luciano, D., Zou, L., and Fosco, J. (2011). Micropayments: A Viable Business Model? Available online at: https://cs.stanford.edu/people/ eroberts/cs181/projects/2010-11/MicropaymentsAndTheNet/solutions.html (accessed February 13, 2021).

Kuśzmierz, B., Müller, S., and Capossele, A. (2021). Committee selection in DAG distributed ledgers and applications. arXiv [Preprint]. Available online at: https: //arxiv.org/abs/2102.03139 (accessed February 17, 2021).

Lambrecht, A., and Skiera, B. (2006). Paying too much and being happy about it: existence, causes, and consequences of tariff-choice biases. J. Mark. Res. 43, 212-223. doi: 10.1509/jmkr.43.2.212

LeMahieu, C. (2018). Nano: A Feeless Distributed Cryptocurrency Network. Available online at: http://media.abnnewswire.net/media/cs/whitepaper/rpt/ 91948-whitepaper.pdf (accessed February 13, 2021).

Noll, D., and Alt, R. (2020). "Internet-of-things marketplaces: state-of-the-art and the role of distributed ledger technology," in Business Information Systems
(BIS 2020), Lecture Notes in Business Information Processing, Vol. 389, eds W. Abramowicz and G. Klein (Cham: Springer), 337-350. doi: 10.1007/978-3-03053337-3_25

Odlyzko, A. (2003). "The case against micropayments," in Financial Cryptography (FC 2003), Lecture Notes in Computer Science, Vol. 2742, ed. R. N. Wright (Berlin: Springer), 77-83. doi: 10.1007/978-3-540-45126-6_6

PayPal (2021). How Can I Update My Payment Preferences for Micropayments? Available online at: https://www.paypal.com/gf/smarthelp/article/how-cani-update-my-payment-preferences-for-micropayments-faq1691 (accessed February 13, 2021).

Popov, S. (2018). The Tangle. Available online at: https://pdfs.semanticscholar. org/5030/1acbdde9e6c57b41fb44be75b9f92276a574.pdf (accessed February 13, 2021).

Popov, S. (2019). IOTA: Feeless and Free. Available online at: https://blockchain. ieee.org/technicalbriefs/january-2019/iota-feeless-and-free (accessed February 13, 2021).

Rooijakkers, E. (2018). GDPR-Compliant Smart Meter Data on the IOTA Tangle: Four Lessons Learned While Putting the Consumer in Control. Available online at: https://medium.com/@erwinrooijakkers/gdpr-compliant-smartmeter-data- on-the-iota-tangle-four-lessons-learned-while-putting-thedea852a5b2aa (accessed February 13, 2021).

Schweizer, A., Knoll, P., Urbach, N., von der Gracht, H. A., and Hardjono, T. (2020). To what extent will blockchain drive the machine economy? Perspectives from a prospective study. IEEE Trans. Eng. Manag. 67, 1169-1183. doi: $10.1109 /$ tem.2020.2979286

Sori, A. A., Golsorkhtabaramiri, M., and Rahmani, A. M. (2020). “Cryptocurrency grade of green; IOTA energy consumption modeling and measurement," in Proceedings of the 2020 IEEE Green Technologies Conference (GreenTech), Lafayette.

Strugar, D., Hussain, R., Mazzara, M., Rivera, V., Lee, J. Y., and Mustafin, R. (2018). "On M2M micropayments: a case study of electric autonomous vehicles," in Proceedings of the IEEE International Conference on iThings, GreenCom, Cyber, CPSCom and SmartData, Atlanta, $1697-1700$.

Toffler, A. (1980). The Third Wave. New York, NY: Morrow.

Turban, E., Outland, J., King, D., Lee, J. K., Liang, T.-P., and Turban, D. C. (eds) (2018). "Electronic commerce payment systems," in Electronic Commerce 2018: A Managerial and Social Network Perspective, (Cham: Springer), 457-499.

World Economic Forum (2020). Redesigning Trust: Blockchain Deployment Kit. Available online at: http://widgets.weforum.org/blockchain-toolkit/pdf/ WEF_Redesigning_Trust_Blockchain_Deployment\%20Toolkit.pdf (accessed February 13, 2021).

Conflict of Interest: The authors declare that the research was conducted in the absence of any commercial or financial relationships that could be construed as a potential conflict of interest.

Copyright (c) 2021 Klein and Stummer. This is an open-access article distributed under the terms of the Creative Commons Attribution License (CC BY). The use, distribution or reproduction in other forums is permitted, provided the original author(s) and the copyright owner(s) are credited and that the original publication in this journal is cited, in accordance with accepted academic practice. No use, distribution or reproduction is permitted which does not comply with these terms. 